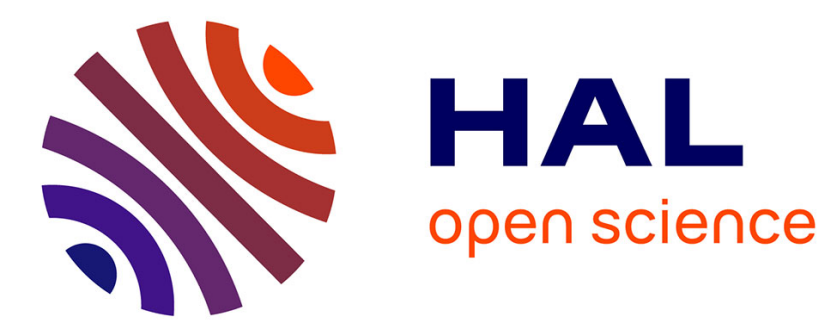

\title{
Non-resonant non-planar free motions of inextensional non-compact beams
}

\author{
Angelo Luongo, Giuseppe Rega, Fabrizio Vestroni
}

\section{To cite this version:}

Angelo Luongo, Giuseppe Rega, Fabrizio Vestroni. Non-resonant non-planar free motions of inextensional non-compact beams. Journal of Sound and Vibration, 1989, 134 (1), pp.73-86. hal-00789390

\section{HAL Id: hal-00789390 \\ https://hal.science/hal-00789390}

Submitted on 18 Feb 2013

HAL is a multi-disciplinary open access archive for the deposit and dissemination of scientific research documents, whether they are published or not. The documents may come from teaching and research institutions in France or abroad, or from public or private research centers.
L'archive ouverte pluridisciplinaire HAL, est destinée au dépôt et à la diffusion de documents scientifiques de niveau recherche, publiés ou non, émanant des établissements d'enseignement et de recherche français ou étrangers, des laboratoires publics ou privés. 


\title{
NON-RESONANT NON-PLANAR FREE MOTIONS OF INEXTENSIONAL NON-COMPACT BEAMS
}

\author{
A. Luongo, G. Rega and F. Vestroni \\ Dipartimento di Ingegneria delle Strutture, delle Acque e del Terreno, Università de L'Aquila, \\ 67040 Monteluco di Roio (AQ), Italy
}

(Received 22 August 1986, and in revised form 14 February 1989)

\begin{abstract}
Non-linear coupling phenomena of non-compact beams with comparable frequencies are studied in the absence of flexural internal resonance. Use is made of approximate, though consistent, partial differential equations which contain the terms of elastic interaction responsible for the stronger non-linear effects involving the torsional component. Upon assuming a one-mode approximation for each spatial variable and applying the Galerkin procedure, three ordinary equations are deduced, and an approximate solution in the absence of all resonances is accomplished via a perturbation technique. An extended investigation of the non-linear coupling is made for beams of open cross-sections with various boundary conditions, by varying some meaningful ratios of the natural frequencies of the beam.
\end{abstract}

\section{INTRODUCTION}

In the past decades a certain number of papers have been devoted to the study of non-planar finite motions of beams [1-8]. Due to the complexity of the description of the problem, various simplifying assumptions regarding the kinematics of the beam and the accounting for the various non-linear elastic and inertia terms have been introduced. Within this ambit it is worth noting that the torsional component has been initially disregarded in the study of the spatial flexural oscillations, restricting the analysis to compact beams for which the torsional frequency is considerably larger than the flexural frequencies.

Only in the work by Crespo da Silva and Glynn $[4,5]$ has the problem been treated rigorously; by referring to the exact kinematics of the beam, general order-three, non-linear equations of motion of inextensional beam were derived in terms of the configuration variables, without neglecting any coupling term among the flexural and torsional components. However, in references [6,7] attention was still focused on compact beams, for which the non-planar motion can be accurately described by two equations in the two flexural displacements only after eliminating the torsional component. Besides, due to weak coupling between flexural motions, only the oscillations in conditions of internal resonance have been studied deeply.

Herein the interest is mainly devoted to the non-planar vibrations of non-compact inextensional beams which exhibit comparable flexural and torsional frequencies; in this case the problem is accurately described by three equations in the two transversal displacements and torsion, all of the same order [9], and strong coupling is induced by the non-linear terms. It is then interesting to study the dynamic phenomena also in the absence of internal resonance. For the free motion of a fixed-free beam an approximate but laborious solution of the complete equations was used in reference [8] to analyze some cases of coupling. In this paper a wider investigation of these phenomena is 
performed; for this purpose, use is made of three simpler equations of motion obtained through a consistent ordering of the mechanical and geometrical parameters of actual beams. They still contain the quadratic and cubic terms of elastic interaction among the flexural and torsional components which are responsible for the stronger non-linear effects. These equations do not contain warping, since its coherent introduction in the non-linear beam model used is far from being straightforward.

Upon assuming a one-mode approximation for each variable of the spatial configuration and applying the Galerkin procedure, a system of ordinary differential equations is obtained, an approximate solution of which is pursued via the perturbation multiple time scale technique [10]. The conditions involving the linear frequencies under which internal resonance occurs at the different orders of the asymptotic solution are examined. Then the solution of the equations in the absence of all resonances is accomplished.

Non-linear coupling phenomena among the three modes are studied for beams with open cross-sections and different boundary conditions, by varying some meaningful ratios of the natural frequencies of the beam and the initial conditions. However, the absence of warping limits the applicability of the solution obtained to some open cross-sections.

\section{EQUATIONS OF MOTION AND PERTURBATION SOLUTION}

In the reference frame $O x_{1} x_{2} x_{3}$, one can consider an inextensional shear-indeformable beam aligned with the $x_{1}$ axis, with length $l$, mass per unit length $m$, torsional mass moment of inertia $I_{1}$, torsional stiffness $E_{1}$, flexural stiffnesses $E_{2}$ and $E_{3}$. The dynamic configuration is described by the angle $\theta(s, t)$ around the axis $x_{1}$ and the transversal displacements $u_{2}(s, t)$ and $u_{3}(s, t), s$ being a curvilinear abscissa and $t$ the time. The non-dimensionalized variables

$$
\tilde{s}=s / l, \quad \tilde{t}=\omega t, \quad \tilde{\theta}=\theta / \rho, \quad \tilde{u}_{i}=u_{i} / l
$$

are introduced, where $\omega$ is any real frequency of the beam and $\rho$ is a scaling factor defined in such a way that the flexural and torsional linearized elastic energies assume the same value when $\tilde{\theta}=\tilde{u}$. In addition, the following relations hold:

$$
\beta_{2}=E_{2} / E_{1}, \quad \beta_{3}=E_{3} / E_{1}, \quad \nu=I_{1} \omega^{2} l^{2} / E_{1}, \quad \mu=m \omega^{2} l^{4} / E_{1} .
$$

Order-three flexural-flexural-torsional equations of the free motion of non-compact beams, obtained from reference [9], are

$$
\begin{gathered}
G_{\theta}=\rho\left\{\nu \rho \ddot{\theta}-\rho \theta^{\prime \prime}-\left(\beta_{2}-\beta_{3}\right) u_{2}^{\prime \prime} u_{3}^{\prime \prime}+\left(\beta_{2}-\beta_{3}\right) \rho\left(u_{2}^{\prime \prime 2}-u_{3}^{\prime \prime 2}\right) \theta\right\}=0, \\
G_{2}^{\prime}=\left\{-\beta_{3} u_{2}^{\prime \prime \prime}+\rho\left(\beta_{2}-\beta_{3}\right)\left(u_{3}^{\prime \prime} \theta\right)^{\prime}-\left(\beta_{2}-\beta_{3}\right) \rho^{2}\left(u_{2}^{\prime \prime \prime} \theta^{2}+2 u_{2}^{\prime \prime} \theta \theta^{\prime}\right)\right\}^{\prime}=\mu \ddot{u}_{2}, \\
G_{3}^{\prime}=\left\{-\beta_{2} u_{3}^{\prime \prime \prime}+\rho\left(\beta_{2}-\beta_{3}\right)\left(u_{2}^{\prime \prime} \theta\right)^{\prime}+\left(\beta_{2}-\beta_{3}\right) \rho^{2}\left(u_{3}^{\prime \prime \prime} \theta^{2}+2 u_{3}^{\prime \prime} \theta \theta^{\prime}\right)\right\}^{\prime}=\mu \ddot{u}_{3},
\end{gathered}
$$

where the tilde has been omitted. The corresponding boundary conditions are

$$
\left.G_{2} \delta u_{2}\right|_{0} ^{1}=0,\left.\quad G_{3} \delta u_{3}\right|_{0} ^{1}=0,\left.\quad H_{\theta} \delta \theta\right|_{0} ^{1}=0,\left.\quad H_{2} \delta u_{2}^{\prime}\right|_{0} ^{1}=0,\left.\quad H_{3} \delta u_{3}^{\prime}\right|_{0} ^{1}=0,
$$

where $G_{2}$ and $G_{3}$ are obtained from equations (3) and

$$
\begin{gathered}
H_{\theta}=\rho^{2} \theta^{\prime}, \quad H_{2}^{*}=\beta_{3} u_{2}^{\prime \prime}-\left(\beta_{2}-\beta_{3}\right) \rho u_{3}^{\prime \prime} \theta+\left(\beta_{2}-\beta_{3}\right) \rho^{2} u_{2}^{\prime \prime} \theta^{2}, \\
H_{3}^{*}=\beta_{2} u_{3}^{\prime \prime}-\left(\beta_{2}-\beta_{3}\right) \rho u_{2}^{\prime \prime} \theta-\left(\beta_{2}-\beta_{3}\right) \rho^{2} u_{3}^{\prime \prime} \theta^{2} .
\end{gathered}
$$

Equations (3) and (4) were obtained from the complete order-three equations of motion through a consistent analysis of the smallness of the terms based on the assumptions of (a) comparable flexural and torsional frequencies of the beam, (b) $\left(\beta_{2}-\beta_{3}\right)$ of order $O(\beta)$, 
which corresponds to the absence of internal resonance between the flexural components. In these conditions the order of the coefficients in the equations is [9]

$$
\beta=O\left(\lambda^{2} / \pi^{2}\right), \quad \mu=O\left(\lambda^{2} \pi^{2}\right), \quad \nu=O\left(\pi^{2}\right), \quad \rho^{2}=O\left(\lambda^{2}\right),
$$

with $\lambda$ being the slenderness of the beam. Since no ordering scheme was introduced on the three configuration variables which still remain of the same order, equations (3) are correct for studying the phenomena of non-linear interaction among transversal and torsional vibrations of beams.

The degree of non-linearity of this problem and the range of amplitudes over which a perturbational procedure provides a satisfactory solution can be evaluated by comparing the orders of the linear, quadratic and cubic terms in equations (3). According to equations (6), they are, respectively,

$$
T^{\mathrm{I}}=O\left(\pi^{2} \lambda^{2} q\right), \quad T^{\mathrm{II}}=O\left(\pi^{2} \lambda^{3} q^{2}\right), \quad T^{\mathrm{III}}=O\left(\pi^{2} \lambda^{4} q^{3}\right),
$$

where $q$ is the amplitude of oscillation. These terms become of comparable magnitude when $q=O(1 / \lambda)$ : that is, for very small values of the amplitudes. This means that the problem is strongly non-linear and the perturbational solution can be correctly utilized in this region of small amplitudes.

It is worth noticing that the problems of either in-plane oscillations or flexural internal resonant motion of inextensional beams already studied in the literature [11,12] are those with weak non-linear behaviour that is very different from the case considered herein. Indeed, in both those cases the non-linear terms are essentially cubic and become comparable with the linear terms for a high amplitude value, $q=O(1 / \pi)$.

A discrete model of the continuum system is obtained by assuming a shape function for each of the three configuration variables $\theta, u_{2}$ and $u_{3}$ and applying the Galerkin procedure. The solution to system (3) is thus sought in the form

$$
\theta=f_{1}(s) q_{1}(t), \quad u_{2}=f_{2}(s) q_{2}(t), \quad u_{3}=f_{3}(s) q_{3}(t),
$$

where the $f(s)$ are the eigenfunctions of the linearized problem and the $q(t)$ are unknown time functions. The integral formulation of the equations of motion (3) and boundary conditions $(4,5)$ is

$$
\begin{aligned}
& \int_{0}^{1}\left\{G_{\theta} \delta \theta+\left(G_{2}^{\prime}-\mu \ddot{u}_{2}\right) \delta u_{2}+\left(G_{3}^{\prime}-\mu \ddot{u}_{3}\right) \delta u_{3}\right\} \mathrm{d} s \\
& \quad+\left.\left(-H_{\theta} \delta \theta-G_{2} \delta u_{2}-G_{3} \delta u_{3}-H_{2}^{*} \delta u_{2}^{\prime}-H_{3}^{*} \delta u_{3}^{\prime}\right)\right|_{0} ^{1}=0 .
\end{aligned}
$$

By integrating by parts, making use of relationships (8) and imposing separate vanishing of terms multiplying $\delta q_{1}, \delta q_{2}$ and $\delta q_{3}$, a system of three ordinary equations of motion with quadratic and cubic non-linearities is obtained:

$$
\begin{gathered}
m_{1} \ddot{q}_{1}+k_{1} q_{1}=-d q_{2} q_{3}-c_{12} q_{1} q_{2}^{2}-c_{13} q_{1} q_{3}^{2}, \\
m_{2} \ddot{q}_{2}+k_{2} q_{2}=-d q_{1} q_{3}-c_{12} q_{1}^{2} q_{2}, \quad m_{3} \ddot{q}_{3}+k_{3} q_{3}=-d q_{1} q_{2}-c_{13} q_{1}^{2} q_{3} .
\end{gathered}
$$

The coefficients in equations (10) depend on the elastic, geometric and inertial properties of the beam through the dimensionless parameters $\rho, \beta, \nu$ and $\mu$ given by equations (1) and (2), and on the integrals of products of the shape functions $f_{i}$. Their explicit expressions are given in Appendix 1.

To obtain the solution to system (10) the multiple scale method [10] is adopted. A perturbation parameter, $\varepsilon$, of the order of the amplitude, is introduced and the variables $q$ are considered functions of a sequence of independent time scales $T_{0}, T_{1}, \ldots, T_{n}$, 
which are related to $t$ by the expressions $T_{n}=\varepsilon^{n} t$. The following expansions of $q_{i}$ in powers of $\varepsilon$ are assumed as solutions to system (10):

$$
q_{i}=\varepsilon q_{i 1}\left(T_{0}, T_{1}, T_{2}\right)+\varepsilon^{2} q_{i 2}\left(T_{0}, T_{1}, T_{2}\right)+\varepsilon^{3} q_{i 3}\left(T_{0}, T_{1}, T_{2}\right)+O\left(\varepsilon^{4} t\right) .
$$

Here three time scales have been considered and the functions $q_{i j}(i, j=1,2,3)$ are assumed to be of order $O(1)$. By expressing the time derivatives in terms of the $T_{n}$ variables, substituting equations (11) into equations (10) and equating coefficients of like powers of $\varepsilon$, a sequence of linear systems with the unknowns $q_{i j}$ is obtained, as follows:

$$
\begin{gathered}
m_{1} D_{00} q_{11}+k_{1} q_{11}=0 \\
\operatorname{order} \varepsilon: \\
\begin{array}{c}
m_{2} D_{00} q_{21}+k_{2} q_{21}=0, \quad m_{3} D_{00} q_{31}+k_{3} q_{31}=0 \\
m_{1} D_{00} q_{12}+k_{1} q_{12}=-d q_{21} q_{31}-2 m_{1} D_{01} q_{11} \\
m_{2} D_{00} q_{22}+k_{2} q_{22}=-d q_{11} q_{31}-2 m_{2} D_{01} q_{21} \\
m_{3} D_{00} q_{32}+k_{3} q_{32}=-d q_{11} q_{21}-2 m_{3} D_{01} q_{31}
\end{array} \\
\begin{array}{c}
\operatorname{order} \varepsilon^{3}: \quad \\
m_{1} D_{00} q_{13}+k_{1} q_{13}=-c_{12} q_{11} q_{21}^{2}-c_{13} q_{11} q_{31}^{2}-d\left(q_{21} q_{32}+q_{31} q_{22}\right) \\
-m_{1}\left(2 D_{01} q_{12}+D_{11} q_{11}+2 D_{02} q_{11}\right)
\end{array} \\
m_{2} D_{00} q_{23}+k_{2} q_{23}=-c_{12} q_{11}^{2} q_{21}-d\left(q_{11} q_{32}+q_{31} q_{12}\right)-m_{2}\left(2 D_{01} q_{22}+D_{11} q_{21}+2 D_{02} q_{21}\right) \\
m_{3} D_{00} q_{33}+k_{3} q_{33}=-c_{13} q_{11}^{2} q_{31}-d\left(q_{11} q_{22}+q_{21} q_{12}\right)-m_{3}\left(2 D_{01} q_{32}+D_{11} q_{31}+2 D_{02} q_{31}\right)
\end{gathered}
$$

Here $D_{i}=\partial / \partial T_{i}$ and $D_{i j}=\partial^{2} / \partial T_{i} \partial T_{j}$. The problem is completed with the initial conditions

$$
q_{j}(0)=\varepsilon q_{j}^{0}, \quad \dot{q}_{j}(0)=\varepsilon \dot{q}_{j}^{0},
$$

$\varepsilon q_{j}^{0}$ and $\varepsilon \dot{q}_{j}^{0}$ being the initial amplitudes and velocities, from which the conditions to be associated with each system of the sequence (12)-(14) are obtained:

$$
\begin{array}{cc}
\text { order } \varepsilon: & q_{j 1}(0)=q_{j}^{0}, \quad D_{0} q_{j 1}(0)=\dot{q}_{j}^{0} ; \\
\text { order } \varepsilon^{2}: & q_{j 2}(0)=0, \quad D_{0} q_{j 2}(0)=-D_{1} q_{j 1}(0) ; \\
\text { order } \varepsilon^{3}: & q_{j 3}(0)=0, \quad D_{0} q_{j 3}(0)=-D_{1} q_{j 2}(0)-D_{2} q_{j 1}(0) .
\end{array}
$$

In the following, the solution of the equations of motion up to the $\varepsilon^{3}$-order is pursued by assuming a generating solution with three non-zero components, in order to study the non-linear coupling phenomena in the absence of internal resonance.

\section{SOLUTION IN THE ABSENCE OF INTERNAL RESONANCE}

The periodic solution

$$
q_{j 1}=A_{j}\left(T_{1}, T_{2}\right) \mathrm{e}^{\mathrm{i} \omega_{j} T_{0}}+\text { c.c. }, \quad j=1,2,3,
$$

is adopted as the solution (generating solution) to system (12). In equations (17), $\omega_{j}=\sqrt{k_{j} / m_{j}}$ is the frequency of the beam over the dimensionless time scale $t, A_{j}\left(T_{1}, T_{2}\right)$ is an unknown complex function and c.c. stands for complex conjugate, the c.c. of $A_{j}$ being denoted with an overbar $\left(\bar{A}_{j}\right)$. The dependence of $A_{j}$ on the time scales $T_{1}$ and $T_{2}$ will be obtained by imposing zeroing of the secular terms at the higher orders of the perturbation solution.

Substitution of equations (17) into the $\varepsilon^{2}$-system (13) gives

$$
D_{00} q_{j 2}+\omega_{j}^{2} q_{j 2}=-d\left[A_{h} A_{k} \mathrm{e}^{\mathrm{i}\left(\omega_{k}+\omega_{h}\right) T_{0}}+\bar{A}_{h} A_{k} \mathrm{e}^{\mathrm{i}\left(\omega_{k}-\omega_{h}\right) T_{0}}\right] / m_{j}-\mathrm{i} 2 \omega_{j} D_{1} A_{j} \mathrm{e}^{\mathrm{i} \omega_{j} T_{0}}+c . c .
$$


In equations (18) and in the following, the indexes $j, h, k$ are different from each other and must be rotated in the clockwise sense starting from the set $1,2,3 ; \delta_{i j}$ is the Kronecker symbol. Analysis of the system shows that internal resonance occurs at the order $\varepsilon^{2}$ if the linear frequencies satsify the conditions

$$
\omega_{j}+\omega_{h}=\omega_{k},
$$

involving all three components $q_{1}, q_{2}$ and $q_{3}$.

In the absence of internal resonance, the zeroing of secular terms in equations (18) gives $A_{j}=A_{j}\left(T_{2}\right)$, showing that no frequency correction occurs at this order. Then, by solving the system and substituting, the equations at the $\varepsilon^{3}$-order are obtained:

$$
\begin{aligned}
D_{00} q_{i 3}+\omega_{j}^{2} q_{j 3}= & -\left\{\sum_{1 i}^{3}\left[d_{j i} A_{j} A_{i}^{2} \mathrm{e}^{\mathrm{i}\left(2 \omega_{i}+\omega_{j}\right) T_{0}}+d_{j i}^{*} \bar{A}_{j} A_{i}^{2} \mathrm{e}^{\mathrm{i}\left(2 \omega_{i}-\omega_{j}\right) T_{0}}\right]\left(1-\delta_{i j}\right)\right. \\
& +d\left[\left(A_{h} B_{k}+A_{k} B_{h}\right) \mathrm{e}^{\mathrm{i}\left(\omega_{k}+\omega_{h}\right) T_{0}}+\left(\bar{A}_{h} B_{k}+A_{k} \bar{B}_{h}\right) \mathrm{e}^{\mathrm{i}\left(\omega_{k}-\omega_{h}\right) T_{0}}\right] \\
& \left.+\left[\sum_{1 i}^{3} \tilde{d}_{j i} A_{j} A_{i} \bar{A}_{i}\left(1-\delta_{i j}\right)+\mathrm{i} 2 k_{j} D_{2} A_{j} / \omega_{j}\right] \mathrm{e}^{\mathrm{i} \omega_{j} T_{0}}\right\} / m_{j}+c . c .
\end{aligned}
$$

In equations (20) the homogeneous solution to system (18) appears, the amplitudes $B_{j}$ of which depend on $T_{1}$ and $T_{2}$. The coefficients $d_{j i}=d_{i j}, d_{j i}^{*}=d_{i j}^{*}, \tilde{d}_{j i}=\tilde{d}_{i j}(i \neq j)$ are given in Appendix 1. Internal resonance at this order occurs when

$$
\omega_{j}=\omega_{k},
$$

involving two components of motion only.

It is worthwhile to notice that even if $A_{j}\left(T_{1}, T_{2}\right) \equiv 0$, and in the absence of any resonance, the $j$ th component is forced dynamically from the other two at the order $\varepsilon^{2}$ provided that these components are both different from zero, this being a phenomenon of non-linear modal coupling. Therefore, a motion with two prevailing components having frequencies dependent on the two non-zero amplitudes $A_{h}$ and $A_{k}$ occurs, in which the third component is of higher order.

Still in the absence of internal resonance, the general solution for $q_{1}, q_{2}$ and $q_{3}$ of the same order is obtained. The zeroing of the secular terms in equations (20), when the polar forms

$$
A_{k}\left(T_{2}\right)=\left[a_{k}\left(T_{2}\right) / 2\right] \mathrm{e}^{\mathrm{i} \varphi_{k}\left(T_{2}\right)}
$$

are introduced and the real and imaginary parts are separated, provides the following differential system with the unknowns $a_{k}, \varphi_{k}$ :

$$
a_{k}^{\prime}=0, \quad \varphi_{k}^{\prime}=\omega_{k}\left[\sum_{1 i}^{3} \tilde{d}_{k i} a_{i}^{2}\left(1-\delta_{k i}\right)\right] / 8 k_{k} .
$$

Here the prime denotes the derivative with respect to $T_{2}$. By integrating equations (23) it follows that

$$
a_{k}=\text { constant, } \quad \varphi_{k}=\hat{\varphi}_{k} t+\varphi_{k}^{0},
$$

where the definition for $T_{2}$ has been accounted for and $\hat{\varphi}_{k}$ depends on the squares of the amplitudes.

Then, by solving equations (20), the functions $q_{j 3}$ are obtained which depend on the amplitudes $C_{j}$ of the homogeneous solution. The latter, as well as $B_{j}$, are assumed to have the same dependence on $T_{1}$ and $T_{2}$ as $A_{j}$. The values of $A_{j}, B_{j}$ and $C_{j}$ at $t=0$ are determined by means of the initial conditions (16) at the different orders; they are given in Appendix 2. 
To write the temporal laws of motion in circular form, the amplitudes $B_{j}$ and $C_{j}$ are rewritten as

$$
B_{j}\left(T_{2}\right)=\left(b_{j} / 2\right) \mathrm{e}^{\mathrm{i} \psi_{j}\left(T_{2}\right)}, \quad C_{j}\left(T_{2}\right)=\left(c_{j} / 2\right) \mathrm{e}^{\mathrm{i} x_{j}\left(T_{2}\right)},
$$

where

$$
\psi_{j}=\hat{\varphi}_{j} t+\psi_{j}^{0}, \quad \chi_{j}=\hat{\varphi}_{j} t+\chi_{j}^{0} .
$$

Substituting $q_{i j}$ in equation (11) and using equations (22) and (25) gives the time laws of motion in circular form as

$$
\begin{aligned}
q_{j}= & \varepsilon a_{j} \cos \Phi_{j}+\varepsilon^{2}\left\{-a_{h} a_{k}\left[\Omega_{j} d \cos \left(\Phi_{h}+\Phi_{k}\right)+\Omega_{j}^{*} d \cos \left(\Phi_{h}-\Phi_{k}\right)\right] / 2+b_{j} k_{j} \cos \Psi_{j}\right\} / k_{j} \\
& +\varepsilon^{3}\left\{-\sum_{1 i}^{3} a_{j} a_{i}^{2}\left[\Omega_{j i} d_{j i} \cos \left(2 \Phi_{i}+\Phi_{j}\right)+\Omega_{j i}^{*} d_{j i}^{*} \cos \left(2 \Phi_{i}-\Phi_{j}\right)\right]\left(1-\delta_{i j}\right) / 4\right. \\
& -a_{h} b_{k}\left[\Omega_{j} d \cos \left(\Psi_{k}+\Phi_{h}\right)+\Omega_{j}^{*} d \cos \left(\Psi_{k}-\Phi_{h}\right)\right] / 2 \\
& \left.-a_{k} b_{h}\left[\Omega_{j} d \cos \left(\Psi_{h}+\Phi_{k}\right)+\Omega_{j}^{*} d \cos \left(\Psi_{h}-\Phi_{k}\right)\right] / 2+c_{j} k_{j} \cos X_{j}\right\} / k_{j} .
\end{aligned}
$$

In equations (27) the dynamic amplification factors $\Omega$ are defined as

$$
\begin{array}{ll}
\Omega_{j}=\omega_{j}^{2} /\left[\omega_{j}^{2}-\left(\omega_{h}+\omega_{k}\right)^{2}\right], & \Omega_{j}^{*}=\omega_{j}^{2} /\left[\omega_{j}^{2}-\left(\omega_{h}-\omega_{k}\right)^{2}\right], \\
\Omega_{i j}=\omega_{i}^{2} /\left[\omega_{i}^{2}-\left(\omega_{i}+2 \omega_{j}\right)^{2}\right], & \Omega_{i j}^{*}=\omega_{i}^{2} /\left[\omega_{i}^{2}-\left(\omega_{i}-2 \omega_{j}\right)^{2}\right]
\end{array}
$$

and the phases are

$$
\Phi_{k}=\bar{\omega}_{k} t+\varphi_{k}^{0}, \quad \Psi_{k}=\bar{\omega}_{k} t+\psi_{k}^{0}, \quad X_{k}=\bar{\omega}_{k} t+\chi_{k}^{0},
$$

$\bar{\omega}_{k}$ being the non-linear frequencies of the three configuration variables:

$$
\bar{\omega}_{k}=\omega_{k}\left[1+\sum_{1 i}^{3} \tilde{d}_{k i} a_{i}^{2} \varepsilon^{2}\left(1-\delta_{k i}\right) / 8 k_{k}\right] .
$$

Equations (27) reveal deep modifications of the temporal laws with respect to the linear ones. Indeed, the motion of the $i$ th component is described by the superposition of several harmonics with frequencies which are combinations of the three non-linear fundamental frequencies (30); namely, $\bar{\omega}_{j}, \bar{\omega}_{h} \pm \bar{\omega}_{k}, 2 \bar{\omega}_{h} \pm \bar{\omega}_{k}(j, h, k=1,2,3 ; h \neq k)$. Harmonics having the same frequency $\left(\bar{\omega}_{j}, \bar{\omega}_{h} \pm \bar{\omega}_{k}\right)$ but different phases occur; however, such differences reduce to zero if the particular case of zero initial velocity for all components is considered.

Equations (30) show that the non-linear frequency of each variable depends on the squares of the amplitudes of oscillation of the remaining two variables.

\section{ANALYTICAL INVESTIGATION}

The analytical investigation is made by using the perturbation solution developed, which gives information on the parameters governing the phenomenon. Due to their large number, in the following the description of the dynamic behaviour of beams is made with reference to specific representative cases selected on the basis of these parameters.

The non-linear dynamic coupling depends both on the values of the ratios among the natural frequencies of the beam and on the amount of coupling occurring already in the static range. Within the assumption of cross-sections with comparable flexural rigidities, the latter is stronger, as these are different from each other, and it is described quantitatively just by the ratio $\beta_{2} / \beta_{3}$ governing the importance of the non-linear terms in the equilibrium equations. Therefore, in the analytical investigation, with reference to open sections with 
torsional rigidity about one hundred times lower than the flexural ones, basically two types of section are considered: (i) with different flexural rigidities $\left(\beta_{2} / \beta_{3}=2 \cdot 60\right)$; (ii) with more nearly equal rigidities $\left(\beta_{2} / \beta_{3}=1 \cdot 19\right)$. The beam frequencies are then varied suitably to analyze the specific effect of the dynamic amplification.

The beams considered have the torsional frequency $\omega_{1}$ greater than the flexural frequencies (with $\omega_{3}>\omega_{2}$ ), as generally occurs in technical applications with open sections. Thus, according to equations (27), the ratios playing the more important role in the dynamic problem are $\left(\omega_{2}+\omega_{3}\right) / \omega_{1}$ and $\omega_{1} / \omega_{3}$ at the quadratic and cubic order, respectively, for section (i), and $\omega_{3} / \omega_{2}$ for section (ii).

Different beams (B1 to B3) are considered with section (i), which is expected to be more interesting. The relevant values of the frequency ratios appearing in the significant dynamic amplification factors (28) are reported in Table 1: they show how the beams are gradually further from a resonant condition involving all three components, and nearer to the resonance between the torsional and one flexural component. In addition, for the sake of comparison, a beam with closed section (BCS) having the same flexural rigidities as the open sections and torsional rigidity of the same order, is also examined. Since in this case the frequencies are not all of the same order, the relevant dynamic results are obtained with the equations of motion in their complete form [8]. In all the other cases the simplified equations (3), specifically derived for treating beams with frequencies of the same order, are referred to. Preliminary investigations on the beams considered, made using the complete and the simplified equations, validated the ordering introduced to obtain the latter equations.

TABLE 1

Frequency ratios of the beams

\begin{tabular}{lcccc}
\hline & BCS & B1 & B2 & B3 \\
\hline$\left(\omega_{2}+\omega_{3}\right) / \omega_{1}$ & 0.14 & 1.19 & 1.35 & 1.48 \\
$\left(2 \omega_{1}-\omega_{3}\right) / \omega_{3}$ & $22 \cdot 2$ & 1.72 & 1.38 & 1.18 \\
\hline
\end{tabular}

In contrast, a unique beam (B4) having section (ii) is considered, with a value of the frequency ratio $\left(2 \omega_{2}-\omega_{3}\right) / \omega_{3}$ appearing in the meaningful dynamic amplification factor equal to $1 \cdot 18$.

Some results showing the non-linear coupling among the three components of motion are now presented, with reference to the time laws, equations (27), which allow insight into the effect of coupling not only in terms of maximum amplitudes. Fixed-free and hinged-hinged beams with different sets of initial conditions (i.c.) are considered, in any case with the assumption of zero initial velocities.

The fixed-free beam (FF) is considered first. The temporal laws for the three components $q_{1}, q_{2}$ and $q_{3}$ obtained for BCS and B1 with zero initial conditions for the twist angle $q_{1}$ and 0.1 for the two flexural variables are plotted in Figures 1 and 2, respectively. The BCS shows a very low torsion $q_{1}$ forced dynamically from $q_{2}$ and $q_{3}$, the amplitudes of these latter components remaining unaltered during the motion; $q_{1 \text { max }}$ reaches only $0 \cdot 16 q_{2 \max }$. Instead, the forced torsional component is much stronger for $\mathrm{B} 1$, with a maximum value equal to $0.90 q_{2 \max }$. This means that the energy content associated with the angle of twist, which is zero initially, increases to a value comparable with that pertaining to a flexural displacement. In addition, B1 also exhibits the effect of coupling between the two flexural variables, due essentially to the forcing effect exerted, in turn, from $q_{1}$. 


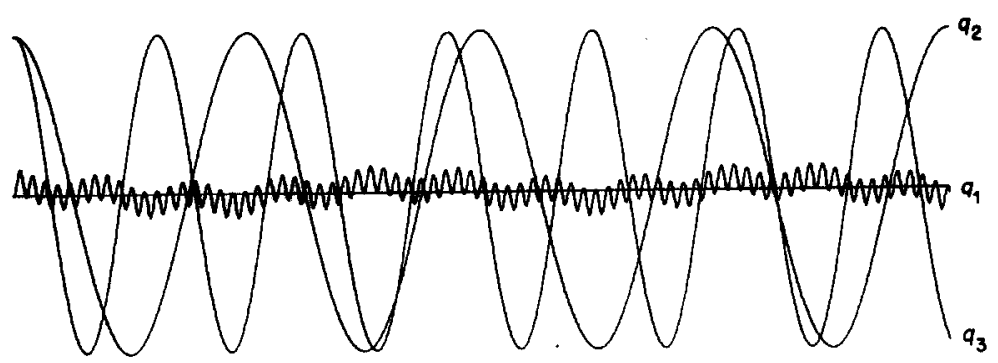

Figure 1. Time laws of the three displacement components for fixed-free beam BCS; $a_{1}, a_{2}, a_{3}=0 \cdot 0,0 \cdot 1,0 \cdot 1$.

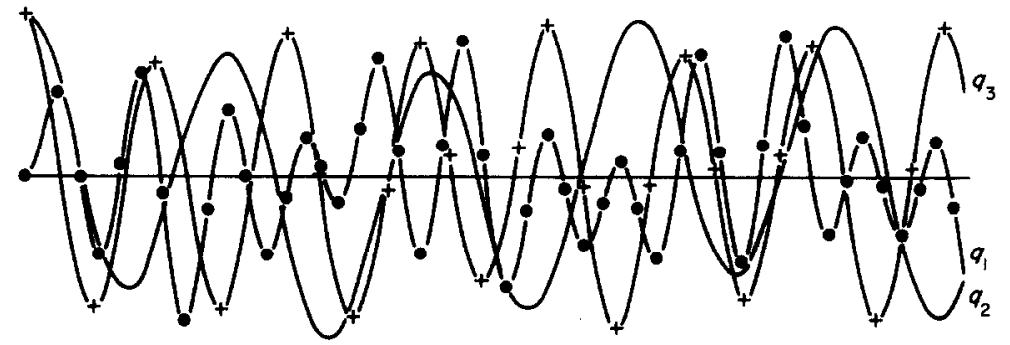

Figure 2. As Figure 1 but for beam B1; $a_{1}, a_{2}, a_{3}=0 \cdot 0,0 \cdot 1,0 \cdot 1$.

The oscillations of B1 obtained with all three initial conditions different from zero are plotted in Figures 3(a) and (b), where the same amplitude of the torsional component is considered but with opposite sign. The results are quite different from each other: indeed, with $a_{1}=0.03$ the non-linear effect on $q_{1 \text { max }}$ is weaker than that obtained in the other case and even weaker than with $a_{1}=0$, while the laws appear less regular and more similar to this last case. The first item is associated with the pattern of the non-linear strain energy of the system,

$$
\Pi=\frac{1}{2}\left(k_{1} q_{1}^{2}+k_{2} q_{2}^{2}+k_{3} q_{3}^{2}\right)+d q_{1} q_{2} q_{3}+c_{12} q_{1}^{2} q_{2}^{2}+c_{13} q_{1}^{2} q_{3}^{2},
$$

calculated at $t=0$, having its minimum (see Figure 4) at a value different from zero
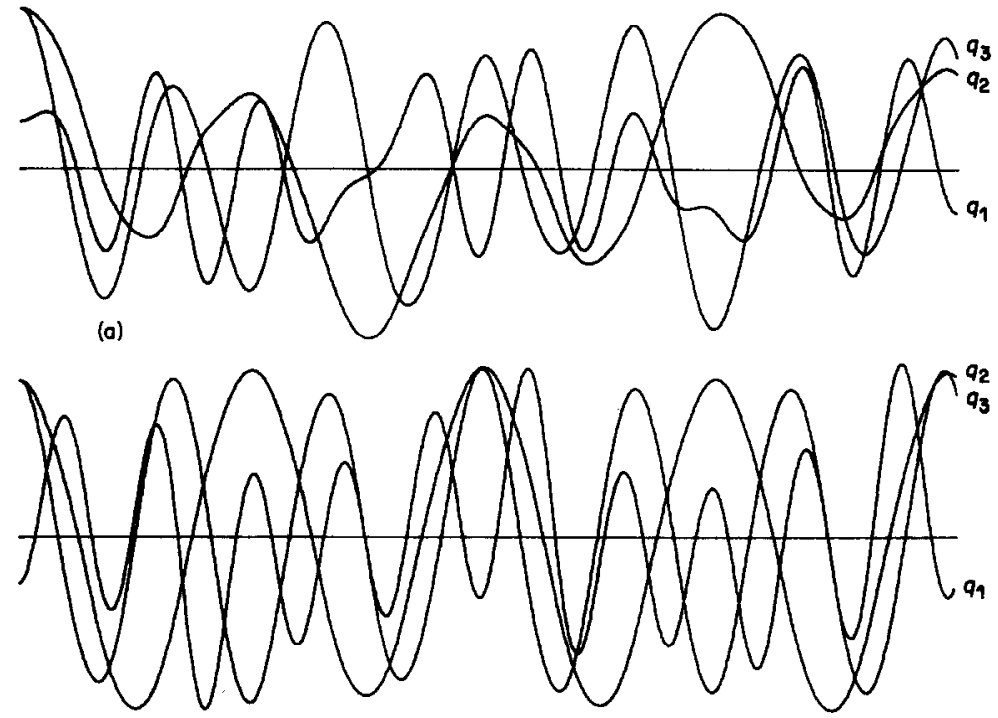

(b)

Figure 3. As Figure 2, but (a) $a_{1}, a_{2}, a_{3}=0 \cdot 03,0 \cdot 1,0 \cdot 1$, and (b) $a_{1}, a_{2}, a_{3}=-0 \cdot 03,0 \cdot 1,0 \cdot 1$. 


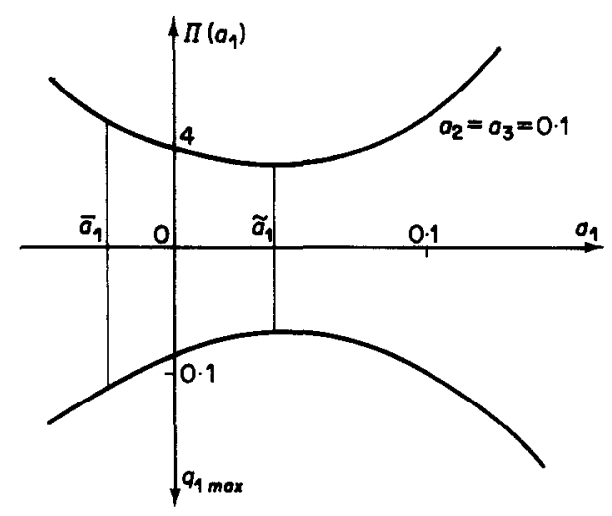

Figure 4. Initial strain energy and maximum response of $q_{1}$ versus $a_{1}$ for assigned values of $a_{2}$ and $a_{3}$. $\left(\tilde{a}_{1}=0.039\right)$, which is related to the static non-linear coupling of the system; correspondingly, the maximum response of $q_{1}$ also attains its minimum at $\tilde{a}_{1}$. The second item, the general regularity of the laws in Figure $3(\mathrm{~b})$, is due to the initial conditions in this case being close to the value $\bar{a}_{1}=-0.027$ for which the harmonic with own frequency does not appear in the $q_{1}$ time law.

The dynamic coupling among the three components is strong for B1 due to proximity to the quadratic resonance condition involving all of them. Indeed, if the results obtained with B2-which is further from that condition-are analyzed, minor coupling effects on the twist angle and therefore between the flexural co-ordinates are observed. Nevertheless, $q_{1 \max }$ still reaches $0.44 q_{2 \max }$, mainly because of static non-linear coupling. The interaction reduces further on passing to $B 3$, the major proximity of which to the resonant condition $\omega_{1}=\omega_{3}$ does not give rise to new coupling phenomena with this set of initial conditions.

The picture is different when finite initial conditions are imposed on torsion and flexure, while the other flexure is either zero or simply perturbed. In Figures 5 and 6 the laws

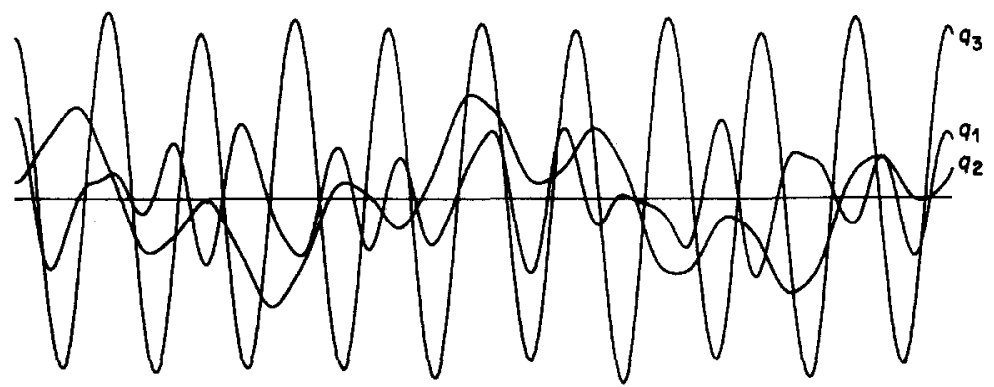

Figure 5. As Figure 2 but for $a_{1}, a_{2}, a_{3}=0.05,0.01,0 \cdot 1$.

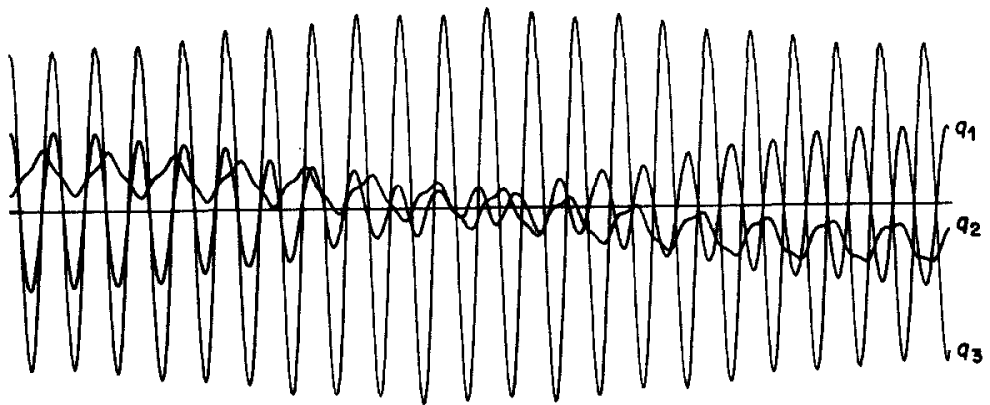

Figure 6. As Figure 5 but for beam B3; $a_{1}, a_{2}, a_{3}=0.05,0 \cdot 01,0 \cdot 1$. 
obtained for B1 and B3 with $a_{1}=0.05, a_{2}=0.01$ and $a_{3}=0.1$ are plotted. For B1, closer to the condition $\omega_{2}+\omega_{3}=\omega_{1}$, the perturbed component $q_{2}$ is notably forced, up to 6.28 times its initial value, and vibrates with a law resulting mainly from the combination of the harmonic of non-linear frequency $\bar{\omega}_{2}$ and that of an amplitude dependent on $a_{1} a_{3}$ and a frequency $\bar{\omega}_{3}-\bar{\omega}_{1}$ almost four times lower. The flexural component $q_{3}$ vibrates with nearly constant amplitude, since only cubic harmonics remain in its law due to smallness of the product $a_{1} a_{2}$. Torsion $q_{1}$ has a complex law resulting from the combination of several harmonics of different amplitudes and frequencies.

B3 exhibits a different response, being closer to the cubic resonance condition $\omega_{1}=\omega_{3}$, involving just the two components which are assigned finite initial amplitudes. A minor forced component $q_{2}$ arises with respect to B1 $\left(q_{2 \max } / a_{2}=3.5\right)$, mainly due to the static coupling. Instead, dynamic interaction and energy exchange occur between $q_{1}$ and $q_{3}$ but to a minor extent relative to those occurring among the three components for $\mathrm{B} 1$, simply because the resonance condition $\omega_{1}=\omega_{3}$ is of cubic order. However, in the case considered, the non-linear frequencies of $q_{1}$ and $q_{3}$ approach each other $\left(\bar{\omega}_{1} / \bar{\omega}_{3}=0.97\right)$, so that the presence of a forced term of frequency $\left(2 \bar{\omega}_{3}-\bar{\omega}_{1}\right) \simeq \bar{\omega}_{1}$ in the first of equations (27) induces beating on $q_{1}$.

It is also interesting to analyze the response of B3 to the set of initial conditions 0.05 , $0 \cdot 1$ and 0.01 (see Figure 7). In this case, in which the almost resonant flexural component $q_{3}$ is simply perturbed, the proximity to the resonance condition $\omega_{1}=\omega_{3}$ does not produce notable coupling effects. Indeed, in the absence of resonance, the interaction between two components can develop only with finite initial values for both of them, while in the condition of resonance it occurs with even small perturbations of one of them.

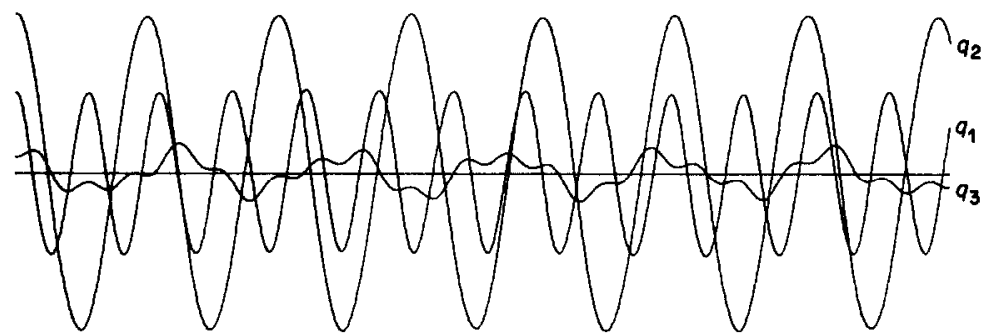

Figure 7. As Figure 6 but $a_{1}, a_{2}, a_{3}=0.05,0 \cdot 1,0.01$.

Consider now the hinged-hinged beam with either one (HH1) or both (HH2) ends torsionally constrained. Analysis of the coefficients of the motion equations (10) reveals the different importance of the non-linear terms with the three boundary conditions considered: namely, they are stronger on passing from the FF beam to $\mathrm{HH} 2$ and $\mathrm{HH} 1$. This is shown by making reference to the case in which non-zero initial amplitudes $\left(a_{2}, a_{3}\right)$ are assigned to the flexural components only. Owing to the non-linear static coupling, the torsional component $a_{1}=\left(-d / k_{1}\right) a_{2} a_{3}$ arises, which in turn gives rise to the main non-linear term in the two flexural equations $\left(d a_{1} a_{i}, i=3,2\right)$. In Figure 8 the ratio between the non-linear and the linear terms in the $j$ th equation $(j=2,3)$ is plotted for beam sections of type (i) in terms of equal flexural amplitudes $\hat{a}_{i}$ normalized in such a way as to have comparable values of the relevant initial energy for the three boundary conditions examined: i.e., $\hat{a}_{i}=a_{i}$ for the FF beam and $\hat{a}_{i}=4 a_{i}$ for the HH beams. Strong differences occur among the three boundary conditions. That occurring between FF and $\mathrm{HH}_{2}$ is associated mainly with the value of the coefficient $d$, which depends on the product of the fiexural and torsional eigenfunctions (see Appendix 1): for the FF beam the former 


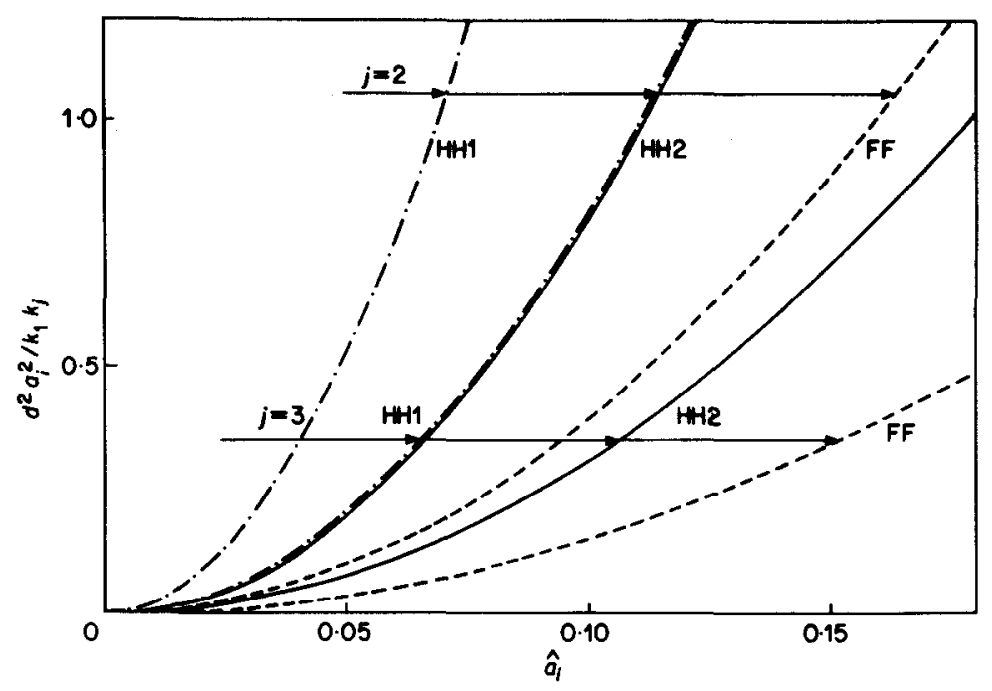

Figure 8. Ratio between non-linear and linear terms in the equations of motion for B1 with different boundary conditions. $\mathrm{FF}, \hat{a}_{i}=a_{i} ; \mathrm{HH}, \hat{a}_{i}=4 a_{i}$.

is maximum where the latter vanishes and vice versa, so that a lower degree of non-linearity occurs. Instead, the further strong difference existing between $\mathrm{HH} 2$ and $\mathrm{HH} 1$ is due mainly to the strong decrease of the torsional rigidity $k_{1}$, which is verified on passing to the HH1 beam.

The different non-linear behaviours of the three beams are shown also in the results of the dynamic analysis performed (see Figure 9 and Table 2). They refer to initial amplitudes $\hat{a}_{2}, \hat{a}_{3}$ for which the ratio between the non-linear and the linear term is less than unity for the beam with stronger non-linear behaviour (HH1), in order that the perturbation solution will furnish accurate results. The non-dimensional time scale considered in Figure 9 is the same for the three beams, which also have equal values of the frequency ratios, so as to evidence directly the effects of non-linearities. The expected stronger non-linear coupling of the HH1 beam comes out clearly from the figure, while Table 2 shows the occurrence of a maximum value of the forced torsional component, becoming more and more large upon passing from $\mathrm{FF}$ to $\mathrm{HH} 2$ to $\mathrm{HH} 1$, as well as higher frequency corrections with respect to the linear values.

The beam of cross-section (ii) with comparable flexural rigidities (B4), which is somewhat closer to the resonance condition $\omega_{2}=\omega_{3}$, should exhibit weaker non-linear behaviour with respect to the beams closer to a resonance condition involving the torsion, as previously noticed in section 2 .

The response of the fixed-free beam is plotted in Figure 10. To account correctly for the major proximity to the flexural resonance condition, it was calculated by using the equations of motion in their complete form [8] rather than equations (10). Use of these latter would lead to an underestimation of the coupling phenomenon already noticeable with the value $\omega_{2} / \omega_{3}=1.09$ considered but of little interest, due to the low degree of non-linearity of this beam confirmed by the analysis of the response. Indeed, notwithstanding higher values of the initial amplitudes, lower interaction between $q_{2}$ and $q_{3}$ occurs than that between $q_{1}$ and $q_{3}$ in the response of B3 (Figure 6), the two beams being equally far from the relevant cubic resonance condition, namely $\omega_{2} \simeq \omega_{3}$ and $\omega_{1} \simeq \omega_{3}$; that is, the former region of resonance is narrower. A not very strong forced torsional component 

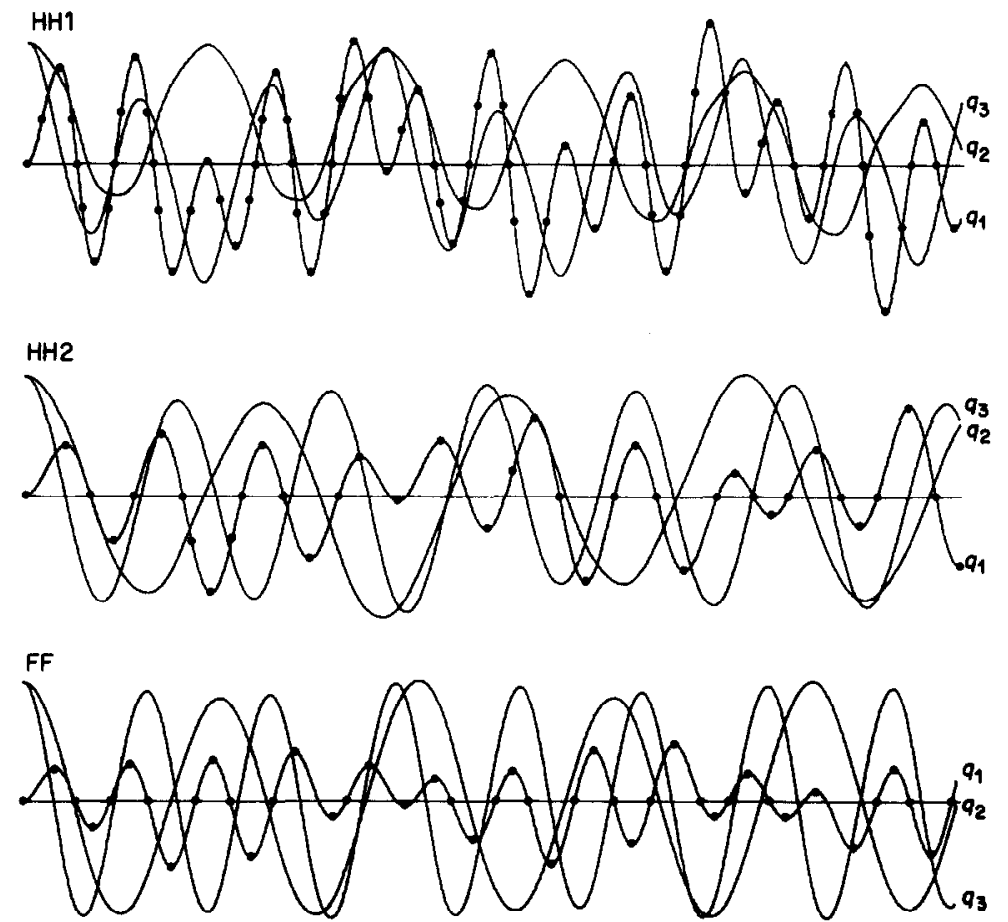

Figure 9. Comparison of non-linear response of beams with different boundary conditions for $a_{1}=0$ and the same flexural amplitudes $\hat{a}_{2}=\hat{a}_{3}=0 \cdot 06$.

TABLE 2

Comparisons of dynamic behaviour of beams $\mathrm{FF}, \mathrm{HH} 2$ and HH1

\begin{tabular}{lcccc}
\hline & $\left|q_{1 \text { max }}\right| /\left|q_{2 \text { max }}\right|$ & $\bar{\omega}_{1} / \omega_{1}$ & $\bar{\omega}_{2} / \omega_{2}$ & $\bar{\omega}_{3} / \omega_{3}$ \\
\hline FF & 0.547 & 0.961 & 1.024 & 1.009 \\
HH2 & 0.796 & 0.919 & 1.050 & 1.019 \\
HH1 & 1.227 & 0.793 & 1.127 & 1.049 \\
\hline
\end{tabular}

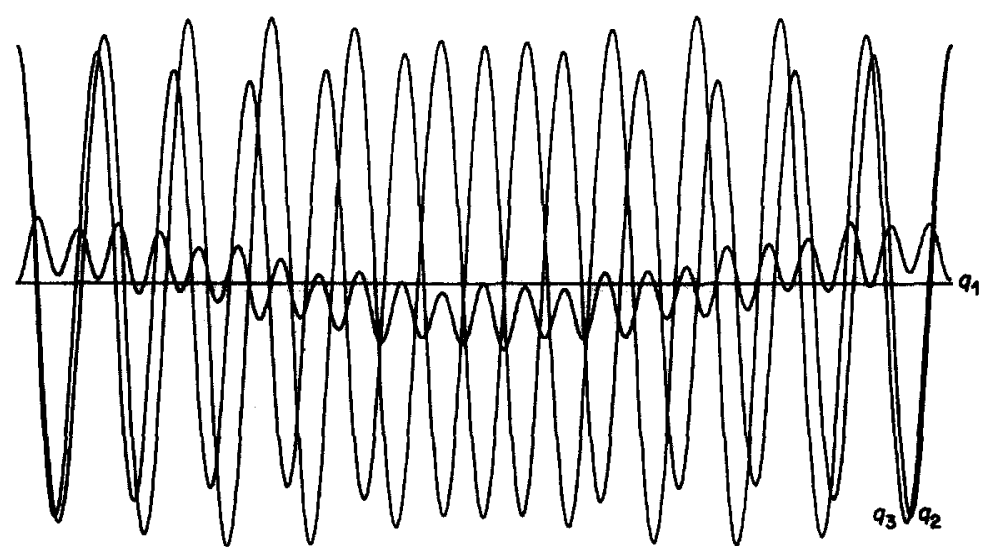

Figure 10. Response of the fixed-free beam B4; $a_{1}, a_{2}, a_{3}=0.0,0.3,0.3$. 
arises in the motion of B4, oscillating with its frequency but modulated on the low time scale by the amplitudes $a_{2}$ and $a_{3}$. Of course, when finite initial conditions are given to torsion and one flexure while the other flexure is simply perturbed, only small forcing effects on this latter co-ordinate are observed.

\section{CONCLUSIONS}

The non-linear dynamic coupling phenomena among flexural and torsional vibrations have been studied for inextensional non-compact beams with all frequencies of the same order and in the absence of internal resonance. Use has been made of accurate equations consistently deduced from more general ones through an ordering of the coefficients, with no assumptions on the configuration variables. The law of motion of the beam has been obtained by means of a third order perturbation analysis.

Some conclusions can be drawn from the investigation performed for beams of open cross-sections with different dynamic characteristics and boundary conditions, selected on the basis of some parameters which govern the phenomenon according to the perturbation solution. It must be underlined that the results presented are obtained within a beam theory in which warping is neglected.

Once the important role played by the non-linear static interaction has been ascertained, the dynamic response is seen to be notably influenced by the values of some meaningful combinations of the linear frequencies, being characterized by different amounts of energy exchange among the co-ordinates. With attention restricted to the combinations involving the torsional frequency-which are the most interesting for analysis within the present context-strong forced torsional motion and associated flexural coupling arise for beams having all comparable frequencies, while very low interaction occurs if the torsional frequency is notably higher than the other ones. In addition, the time response changes notably depending on the resonance condition which is approached and the initial amplitudes considered.

In summary, when a resonance condition involving three components-which is of quadratic order-is approached, even though one component is assigned a zero initial value, it is forced strongly in the motion and in turn modifies the laws of the remaining two. Instead, when a condition-of cubic order-involving two components is approached, and provided that they are given finite initial amplitudes, notable although lower energy exchange occurs between them, while the third component is forced slightly if it is zero or simply perturbed initially; in particular, little interaction occurs where the condition does not involve the torsional frequency.

\section{ACKNOWLEDGMENT}

This research was partially supported by MPI $40 \%$ funds.

\section{REFERENCES}

1. E. C. HAIGHT and W. W. KING 1971 Journal of the Acoustical Society of America 52, 899-911. Stability of nonlinear oscillations of an elastic rod.

2. C. H. Ho, R. A. SCOTT and J. C. EISLEY 1975 International Journal of Non-linear Mechanics 9, 113-127. Nonplanar, nonlinear vibrations of a beam-I: forced motions.

3. C. H. Ho, R. A. SCOTT and J. C. Eisley 1976 Journal of Sound and Vibration 47, 333-339. Nonplanar, nonlinear vibrations of a beam-II: free motions.

4. C. C. GLYNN and M. R. M. CRESPO DA Silva 1977 Report No. ES.77-112 Department of Engineering Science, University of Cincinnati, Cincinnati, Ohio 45221. Non-planar, non-linear oscillations of an elastic rod. 
5. M. R. M. CReSPO DA SILVA and C. C. GLYNN 1978 Journal of Structural Mechanics 6, 437-448. Non-linear flexural-flexural-torsional dynamics of inextensional beams: I. Equations of motion.

6. M. R. M. CRESPO DA SILVA and C. C. GLYNN 1978 Journal of Structural Mechanics 6, 449-461. Non-linear flexural-flexural-torsional dynamics of inextensional beams: II. Forced motions.

7. M. R. M. CRESPO DA SILVA and C. C. GLYNN 1979 International Journal of Solids and Structures 15, 209-219. Non-linear non-planar resonant oscillations in fixed-free beams with support asymmetry.

8. A. Luongo, G. RegA and F. Vestroni 1984 Proceedings of the 7 th Italian Congress of Theoretical and Applied Mechanics V, 169-180. Nonlinear nonresonant flexural-flexuraltorsional motions of inextensional beams.

9. A. LuONGO, G. REGA and F. VeSTRONI 1986 Report No. 106, Istituto di Scienza delle Costruzioni, Università de L'Aquila. Nonplanar finite dynamics of inextensional beams.

10. A. H. NAYFEH 1981 Introduction to Perturbation Techniques. New York: John Wiley.

11. S. ATLURI 1973 Journal of Applied Mechanics 40, 121-126. Nonlinear vibration of hinged beam including nonlinear inertia effects.

12. A. LuONGO, G. ReGA and F. VeSTRONI 1986 Journal of Applied Mechanics 53, 619-624. On nonlinear dynamics of planar shear-indeformable beams.

APPENDIX 1: EXPRESSIONS FOR THE COEFFICIENTS $m, k, c, d$

$$
\begin{gathered}
m_{1}=\nu \rho^{2} \int_{0}^{1} f_{1}^{2} \mathrm{~d} s, \quad m_{2}=\mu \int_{0}^{1} f_{2}^{2} \mathrm{~d} s, \quad m_{3}=\mu \int_{0}^{1} f_{3}^{2} \mathrm{~d} s, \\
k_{1}=\rho^{2} \int_{0}^{1} f_{1}^{\prime 2} \mathrm{~d} s, \quad k_{2}=\beta_{3} \int_{0}^{1} f_{2}^{\prime \prime 2} \mathrm{~d} s, \quad k_{3}=\beta_{2} \int_{0}^{1} f_{3}^{\prime \prime 2} \mathrm{~d} s, \\
d=-\rho\left(\beta_{2}-\beta_{3}\right) \int_{0}^{1} f_{1} f_{2}^{\prime \prime} f_{3}^{\prime \prime} \mathrm{d} s, \quad c_{12}=\rho^{2}\left(\beta_{2}-\beta_{3}\right) \int_{0}^{1} f_{1}^{2} f_{2}^{\prime 2} \mathrm{~d} s, \\
c_{13}=-\rho^{2}\left(\beta_{2}-\beta_{3}\right) \int_{0}^{1} f_{1}^{2} f_{3}^{\prime \prime 2} \mathrm{~d} s, \\
d_{12}=\left(c_{12}-\Omega_{3} d^{2} / k_{3}\right), \quad d_{12}^{*}=\left(c_{12}-\Omega_{3}^{*} d^{2} / k_{3}\right), \quad \tilde{d}_{12}=d_{12}+d_{12}^{*}, \\
d_{13}=\left(c_{13}-\Omega_{2} d^{2} / k_{2}\right), \quad d_{13}^{*}=\left(c_{13}-\Omega_{2}^{*} d^{2} / k_{2}\right), \quad \tilde{d}_{13}=d_{13}+d_{13}^{*}, \\
d_{23}=-\Omega_{1} d^{2} / k_{1}, \quad d_{23}^{*}=-\Omega_{1}^{*} d^{2} / k_{1}, \quad \tilde{d}_{23}=d_{23}+d_{23}^{*} .
\end{gathered}
$$

APPENDIX 2: EXPRESSIONS FOR THE INITIAL VALUES OF THE AMPLITUDES $\boldsymbol{A}_{j}$, $B_{j}, C_{j}$ OF THE HOMOGENEOUS SOLUTION

$$
\begin{aligned}
& A_{j}(0)=\frac{1}{2}\left(q_{j}^{0}-\mathrm{i} \dot{q}_{j}^{0} / \omega_{j}\right), \\
& B_{j}(0)=\left(1 / 2 k_{j}\right)\left\{\left[\Omega_{j} d\left(A_{h} A_{k}+\bar{A}_{h} \bar{A}_{k}\right)+\Omega_{j}^{*} d\left(\bar{A}_{h} A_{k}+A_{h} \bar{A}_{k}\right)\right]\right. \\
&\left.+\left(1 / \omega_{j}\right)\left[\left(\omega_{h}+\omega_{k}\right) \Omega_{j} d\left(A_{h} A_{k}-\bar{A}_{h} \bar{A}_{k}\right)+\left(\omega_{k}-\omega_{h}\right) \Omega_{j}^{*} d\left(\bar{A}_{h} A_{k}-A_{h} \bar{A}_{k}\right)\right]\right\}_{0}, \\
& C_{j}(0)=\left(1 / 2 k_{j}\right)\left\{\Omega_{j h} d_{j h}\left(A_{j} A_{h}^{2}+\bar{A}_{j} \bar{A}_{h}^{2}\right)+\Omega_{j h}^{*} d_{j h}^{*}\left(\bar{A}_{j} A_{h}^{2}+A_{j} \bar{A}_{h}^{2}\right)+\Omega_{j k} d_{j k}\left(A_{j} A_{k}^{2}+\bar{A}_{j} \bar{A}_{k}^{2}\right)\right. \\
&+\Omega_{j k}^{*} d \bar{d}_{j k}^{*}\left(\bar{A}_{j} A_{k}^{2}+A_{j} \bar{A}_{k}^{2}\right)+\Omega_{j} d\left(A_{h} B_{k}+A_{k} B_{h}+\bar{A}_{h} \bar{B}_{k}+\bar{A}_{k} \bar{B}_{h}\right) \\
&+\Omega_{j}^{*} d\left(\bar{A}_{h} B_{k}+A_{k} \bar{B}_{h}+A_{h} \bar{B}_{k}+\bar{A}_{k} B_{h}\right)+\mathrm{i}\left(D_{2} A_{j}+D_{2} \bar{A}_{j}\right) k_{j} / \omega_{j} \\
&+\left[\left(\omega_{j}+2 \omega_{h}\right) \Omega_{j h} d_{j h}\left(A_{j} A_{h}^{2}-\bar{A}_{j} \bar{A}_{h}^{2}\right)+\left(2 \omega_{h}-\omega_{j}\right) \Omega_{j h}^{*} d{ }_{j h}^{*}\left(\bar{A}_{j} A_{h}^{2}-A_{j} \bar{A}_{h}^{2}\right)\right. \\
&+\left(\omega_{j}+2 \omega_{k}\right) \Omega_{j k} d_{j k}\left(A_{j} A_{k}^{2}-\bar{A}_{j} \bar{A}_{k}^{2}\right)+\left(2 \omega_{k}-\omega_{j}\right) \Omega_{j k}^{*} d d_{j k}^{*}\left(\bar{A}_{j} A_{k}^{2}-A_{j} \bar{A}_{k}^{2}\right) \\
&+\left(\omega_{h}+\omega_{k}\right) \Omega_{j} d\left(A_{h} B_{k}+A_{k} B_{h}-\bar{A}_{h} \bar{B}_{k}-\bar{A}_{k} \bar{B}_{h}\right) \\
&\left.\left.+\left(\omega_{k}-\omega_{h}\right) \Omega_{j}^{*} d\left(\bar{A}_{h} B_{k}+A_{k} \bar{B}_{h}-A_{h} \bar{B}_{k}-\bar{A}_{k} B_{h}\right)\right] / \omega_{j}\right\}_{0} .
\end{aligned}
$$

